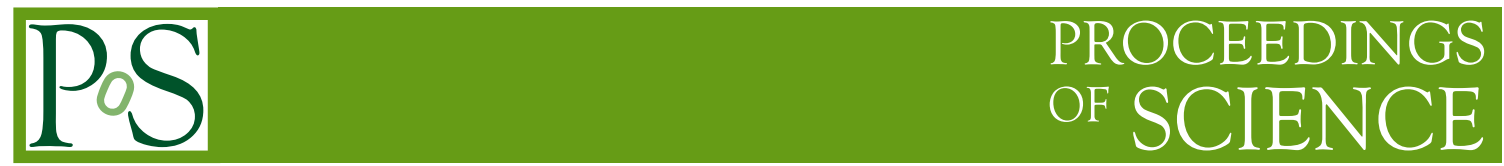

\title{
Small Representation Explaing, Why Standard Model Group
}

\section{Holger Bech Nielsen*}

Niels Bohr Institutet, Blegdamsvej 15 -21 DK 2100Copenhagen

E-mail: hbech@nbi.dk, hbechnbi@gmail.com

The main point is the finding, that properly defined the Standard Model Gauge Group $S(U(2) \times$ $U(3))$ as developed through hard phenomenological work historically can be characterized as that Lie group, which has the minimal size faithfull representation(roughly). At the end I argue for the scenario that the Standard Model will remain valid almost all the way to the Planck scale except for some "see saw neutrinoes".

Proceedings of the Corfu Summer Institute 2014 "School and Workshops on Elementary Particle Physics and Gravity",

3-21 September 2014

Corfu, Greece

\footnotetext{
* Speaker.
} 
Continuation of work with Don Bennett on "What is special about the Standard Model Group" $[2,1]$ presented by Holger Bech Nielsen. Seeking to extract the information as estimated by Svend Erik Rugh, Syrlykke and HBN[11] yet unexplained for the Standard Model Group.

\section{Introduction}

To day we are in the situation that the highest accelator enrgies reprsented by LHC just confirms the at the present scale best theory, the Standard Model, by not finding so far any new fundamental particles - especially not the much hoped for supersymmetry partners -, and so we may have to face the possibility that there is very long to the new physics! Such a situation would call for understanding the problems with such pure Standard Model scenario, i.e.

- 1. Is it satisfactory to have a theory that is only renormalizable, but does not give in principle finite results?

- 2. Including also gravity can we even get a renormalizable theory? One thinks at least something new has to happen when approaching the Planck scale.

- 3. Neutrino-oscillations at least seems to require some new physics, at say the see-saw neutrino scale.

- 4. Can we understand the dark matter (as essentially "seen" astronomically) as comming from the Standard Model. Here it is that I and my collaborators contrary to most other physicists would like to answer: Yes we can imagine dark matter being some pearl sized balls with the enormous mass of about $10^{8} \mathrm{~kg}(=100000 \mathrm{ton})$, which can be understood in terms of a new vacuum which results from just Standard Model with no new physics fundamental fields. Only bound states - of 6 top +6 anti top quarks - and condensates of such bound states are needed, but no new fundamental particles.[5, 36]

- 5. Do we not need a special inflaton field, $r$ can we use the Higgs field. With only Standard Model we should use in principle only the Higgs field, or perhaps some mysterious field for a bound state comming from the Standard Model.

- 6. We must understand also the problem with the Standard Model that if we had that alone, the anomaly for conservation of lepton and baryon numbers would have meant, that the phenomenologically observed excess of matter over antimatter, which is witnessed even by our own existence, would have been washed away. Presumably the see-saw neutrino physics needed for neutrino oscillations anyway would be able to take care of this problem.

- 7. The fine tuning problems would also have to be understood. Here it is that it is the attitude of the present article and of several earlier works of ours that we shall choose rather than to really "solve" the finetuning problems by finding some clever symmetry that can explain them,we shal truly postulate just an as simple as possible finetuning law or rule. This fine tuning rule, which we propose is what we call Multiple Point Principle[37] and it says that the coupling constants are finetuned so as to arrange that there are several - in pracsis below 
three - vacua with very small, meaning of the order of the dark energy in the universe as observed astronomically, energy densities. Each time a vacuum energy density is fixed to be small / essentially zero a coupling constant (combination) gets finetuned. If we have luck with this as I shall review, then we may solve three finetuning problems by three vacua beig imposed small energy densities[38, 37, 12].

There are many things I would like to tell about such as:

- How we might get almost all the structure of the Standard Model by requiring in a very sepecial way that representations (of the particles) be the smallest ones[2, 1, 4]! (Earlier I worked with Niels Brene on an alternative idea also seeking to characterize the Standard Model group [10].)

- How we might escape most of the arguments for there being "new physics", so we could in fact take the message from LHC so far seriously (but this message is not yet there) : that there seems to be no new physics (even I must though admit that we cannot escape neutrinooscillations form signaling new physics).

- But at least for dark matter [36, 5]we have a proposal inside the Standard Model although in a slightly complicated model with bound state of 6 top +6 anti tops and a new vacuum with a Boson condensate of such bound states. Balls made from material much like white dwarf-star-stuff, but surrounded by a skin seperating a vacuum phase with the bound state condensate from a phase without this condesate and of the size like a pearl and a weight $10^{8}$ $\mathrm{kg}$ make up the dark matter (about one astronmic unit between one ball and the next.). One such ball fell in Tunguska in 1908 .

- I and Don Bennett invented a "game" $[2,1]$ between possible Gauge groups so that the Standard Model Gauge group [3] $S(U(2) \times U(3))$ (with respect to the Lie algebra equivalent to $U(1) \times S U(2) \times S U(3))$ wins.

- How we at least - Colin Froggat and I - have a chance of proposing a model for dark matter $[36,5]$ inside the Standard Model in a complicated way, so that we could imagine that the Standard Model is the final answer for very long up in energy(almost to the Planck scale except for some see-saw neutrinoes).

\section{Smallest Volume of a Faithful Represntation Compared to that of the "Adjoint" Representation}

Crudely the main point is this:

- All Lie groups (potential gauge groups for the right model for Nature) have representations, and are properly represented by the faithful representations.

- It is very natural, although slightly convention dependent, to define a volume for the image of a Lie group being mapped into a representation using a "natural" distance concept:

$$
d s^{2}=\frac{1}{d i m} * \operatorname{Tr}\left(|(\mathbf{U}(g)-\mathbf{U}(g+d g))|^{2}\right),
$$


where $\mathbf{U}(g)$ is a (unitary) representation of the group element $g$ and $g+d g$ symbolizes an hereto infinitesimally close element, while the numerical value symbol $|\ldots|$ is taken for the matrices to be defined as $|A|^{2}=A A^{\dagger}$. The number dim is the dimension of the representation matrices $\mathbf{U}(g)$.

- Even for Lie groups with Abelian components we may construct a replacement for an adjoint representaton, let us say "Adjoint"(now in quotation marks).

- Then the Standard Model Gauge group "wins" by having the smallest represetation volume for a faithful representation compared to the volume of the "Adjoint" representation for the group.

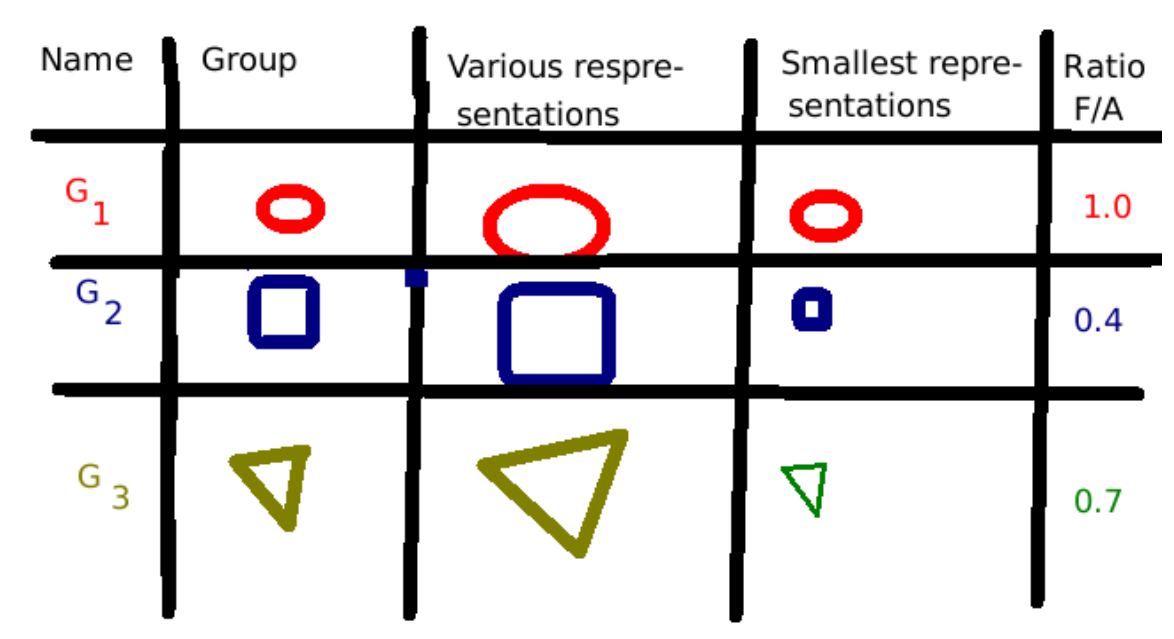

\section{Explanation of the Symbolic Figure with the Groups and their Representations}

- Using the metric

$$
d s^{2}=\frac{1}{\operatorname{dim}} * \operatorname{Tr}\left(|(\mathbf{U}(g)-\mathbf{U}(g+d g))|^{2}\right),
$$

the representation copies of the group look very similar (the same for simple groups), only/mainly the size of these images ( $=$ the different faithfull representations) are different. (Truly a faithfull representation considered as Riemannian manifold imbedded into the space of unitary matries of order dim is in correspondance with the group itself and has the (inner) geometry metric which only deviates from that on the group by a numerical factor bing the same all along the manifold; but this is true only provided one keeps to only count distances along the manifolds)

- Typically any group has aritrarily large representations measured in the metric.

- But there is a lower limit to the size of the faithfull representations (while non-faithfull ones count also the trivial representation, which in some sense is infinitely small, although we might argue it to have indetermined size; the point is that the purely zero representation can be said to have dimension of the representation $\operatorname{dim}=0$ and so the distances become $0 / 0=$ "ill defined") 
- By comparing any representation size - say the volume taken to the (1/"group dimension")th power - to the "Adjoint" (slightly generalized) we can compare representations even for different groups .

\section{The crux of our principle:}

Nature so loved small (faithfull) representations, that it even selected the gauge group of the true model (= the Standard Model) so that the gauge group could have the smallest - relative to the "adjoint"(slightly generalized) - possible faithfull representation whatsoever!

(analogue style to Johannes 3.14)

\section{Selecting Goal Quantity}

- We want to use the volume of the representation relative to that of the "adjoint" representation.

- We want to choose the "goal quantity" ( = "the score") so as to be balanced w.r.t. dimension of the (gauge) group being tested; therefore we want to take the $d_{G}$ th root of the volume, so that it becomes rather say the linear scale size ratio. (Here $d_{G}$ is the dimensionality of the Lie group $G$.) By this being balanced w.r.t. dimension we have in mind that we should avoid that "goal quantity" would have so much dependence on the dimension of the Lie group that it would make this dimension selcted rather than some hoped for more dimension independent stucture. As a sign of such a balance having been at least attempted is that the goal quantity is arranged to be the same for a group $G$ and for any cross product of this group with itself $G \times G \times G \times \cdots \times G$

- For quite conventional and accidental reasons we started to consider the inverse square of the linear - understood in the metric - size of the "the image of smallest faithful representation $F$ " compared to the same linear size of the "adjoint representation" $A$.

This means that we look at

$$
\text { "goal quantity" }=\left(\frac{\operatorname{Vol}(F)}{\operatorname{Vol}(A)}\right)^{-2 / d_{G}}=\left(\frac{\operatorname{Vol}(A)}{\operatorname{Vol}(F)}\right)^{2 / d_{G}}
$$

\subsection{Reminder of the main motivation for the whole game with this "goal quantity"}

Let us remind the reader about why this "goal quantity" is so important: The by Nature chosen gauge group- namely the Standad Model group $S(U(2) \times U(3)$ ) (which has the same Lie algebra as the Lie algebra of the Standard Model $\mathbf{R} \oplus \mathfrak{u n}(2) \oplus \mathfrak{l n}(3))$ has the smallest "goal quantity" (if one uses the definition just above; but one should ask for the biggest if one took a definition being like the one in earlier paper an inverse or inverse square of the present one of course):Thus it selects or explains the Standard Model group.

Let us even mention that requiring the minimal "goal quantity" for the Lorentz-group taken for simplicity as the compact one $S O(d)$ for $d$ dimensional space-time leads to the "winning" dimension being $d=3$ or $d=4$ and thus our goal quantity puts itself into the series of explanations 
attempted to be given to the question: Why do we have just $3+1$ space time dimensions $[1,7,8,6]$. Getting only to an equal race between 3 and 4 space time dimensions is of course not quite as impressive as if 4 (the phenomenological value) had been sigled out alone. In the article [1] I did invent some "improvements" taking crudely the whole Poincare group into account and managed by such compliation - even if a bit motivated, somewhat less beuatyfull - to make the $4=3+1$ to win.

\subsection{Special problems with the Abelian invariant subalgebras:}

- A priori there are no adjoint representation to compare with for Abelian groups.

- A priori an Abelian Lie group has a continuum of different representations - all the irreducible ones are one dimensional representations - namely having different charges $e$ (meaning a representation by a $1 \times 1$ matrix $\exp (i e \delta)$ where the group element is $\exp (i \delta) \in U(1))$

- The charges get only quantized, when one considers a compactified Lie group $U(1)$ rather than $\mathbf{R}$.

\section{Treatment of Abelian Part}

The easiest way to treat the Abelian part:

- Have in mind that working with groups rather than just Lie algebras - which is the only thing that matters for the Yang-Mills gauge field couplings - O'Raifeartaigh [3] defines the significance of the group from its restrictions on the matter field representations. (It might be good to think of this type of restrictions as generalizations of the quantitzation of charge obtained from the existence of (Dirac) monopoles. In fact David Olive found the monopole that can just deliver the restriction for the Standard Model [13].)

- A short way is then to define the factor in the "volume of the representation $r$ compared to that of the replacement for the adjoint representation" formally by means the system of allowed representation under the group $G$ in question.

- Indeed the restriction from the group rather than only from the Lie algebra is given by a (charge) on which representations are allowed may be described by what we call a "quantization rule". Such a quantization rule is the restriction on the representations for the Lie algebra which would select out those representations allowed as representations of the group. E.g. in the case of the Standard Model group $S(U(2) \times U(3))$ the quantization rule reads

$$
Y / 2+I_{W 3}+" \text { triality" } / 3=0(\bmod 1)
$$

where $I_{W 3}$ is the third component of the weak isospin, and $Y$ the weak hypercharge, so that the electric charge is $Q=Y / 2+I_{W 3}$. Further "triality" $/ 3$ is $1 / 3$ for quarks $-1 / 3$ for anti quarks, 0 for gluons. (Note that for the Standard Model this "quantization rule" is just the extension to the inclusion of the quarks with their - somewhat mysterious - of the simple Millikan[14] rule of charge quantization(charge being an integer multiplum of the charge quantum). 
- Take the ratio of the "number" of allowed representations of the maximal Abelian invariant subalgebra, when no restrictions is put to which representations for the non-abelian invariant subgroups we use. Then compare that to the "number" of those charge combinations, which are allowed, if the representations of the non-abelian invariant subgroups are required trivial. (Really there are infintely many of both two types of charge combinations mentioned here, but it is not so difficult to define a meaningful and finte ratio of the two infinite "numbers")

- The number of allowed representations of both kinds are typically infnite, but one may take a limit, and then this ratio is the extra factor to put on the volume ratio for the non-abelian part as the contribution from the abelian part.At the end one then takes the "total dimension"th root and the minus second power to get to our "goal quantity".

\section{Noting Philosophy of Extracting the Teaching of Nature from Structure of the Gauge Group}

The reader should have in mind that we physicists having only accelerators like even LHC with very small energies per elementary particle compared to say the Planck scale, where the really true new physics may show up. Thus we would love, if we could guess from some very pregnant regularity being discovered at our "low" (compared to Planck scale) energy something about the Planck scale or just somewhat higher up in energy scale physics. This means we dream about being as lucky as Dalton [19] was, when he could argue for the existence of molecules/atoms from the simple rational volume ratios between gasses, that made chemical reactions (e.g. oxygen burning with hydrogen to make only water in a very simple rational ratio of the volumes: two parts hydrogen to one part oxygen). But such a simple rule, so simple that we can trust its suggestion, does not have only to be concerning numbers, no, it could also concern structure, such a selecting a gauge group. The important thing is just that the number of reasonably comparably simple possibilities among which nature has selected the so remarkable one, is sufficiently high. It were these numbers of possibilities that were presented in our paper by Rugh et al. [11] in the form of giving the number of digital cifers needed to specify among similarly reasonable choices just that one that Nature has chosen. We[11] Rugh et al. called this number of cifers needed the amount of information not yet explained measured in bits. If we make a model, that in this language explains a large number of bits, it almost must be true, but if it only explains a small number of bits it could easily be just accidentally "a remarkable choice of nature".

\section{The main point of the present talk is:}

To make Nature give us a hint towards the true model beyond the Standard Model by seeking - rather unbiased - a relatively simple property characterizing just the Standard Model Group $S(U(2) \times U(3))$ ( Lie-algebra-wise equivalent to $U(1) \times S U(2) \times S U(3)$ ). It will turn out that I can use almost the same idea to characterize the phenomenological dimension $d=4$ [1] (dimension were also explained much earlier [6,7], and even for telling the representations of the Fermions[18, 4] and the Higgs .

But really the amount of information in the not explained gauge group of the Standard Model is only -according to our, i.e. Rugh et al's, estimate 8 bits (hardly two letters) while the dimension 
$d=4$ gives $\approx 3$ bits, i.e. about a half letter. Nevertheless we shall now seek to get some usefull information out of the "book of nature" this way.

\section{Rughs et al.'s Information Counting; Not Explained Information.[11]}

\begin{tabular}{|l|l|l|}
\hline $\begin{array}{l}\text { Unexplained } \\
\text { information }\end{array}$ & $\begin{array}{l}\text { Amount in } \\
\text { bits }\end{array}$ & \\
\hline Parameters & $\mathbf{1 4 3}$ to 155 & \\
\hline $\begin{array}{l}\text { Structural Information } \\
\text { divided out as: }\end{array}$ & $\mathbf{2 1}$ & \\
\hline Gauge group & 8 & \\
3+1 dimensions & 3 & \\
Spin-distribution & 6 & \\
Higgs-representation & 4 & \\
\hline
\end{tabular}

The information (not explained) in the parameters of the Standard Model making up ca 150 bits is like the information in 30 letters, and the 21 bits in the structure of Standard Model is like 4 letters. In all a half line. (Information in the Weyl-representations $\approx 92$ bits have been counted as explained, but that may be exagerated? The idea we refer to here is this: If you assume that we can only hope to observe fermions, which are mass protected by the gauge charges in the Standard Model because "you" (= the physicists ) are so "poor" that we only have energies very small compared to the presumed fundamental scale at our disposal per particle, then there can only be fermions of one handedness, when there exist non of the opposite one with the same gauge charge quantum numbers. This requirement restricts of course the system of fermions a lot, and thus gives/explains a lot of information. It is this information/explanation which we refered to as the 92 bits)

\section{More Precise Strategy:}

Don Bennett and I found - by complicated calculations and speculations, which I shall not tell you about now - a quantity depending on/defined for groups, or at first rather Lie algebras,

$$
=\left(\prod_{\text {simple groups } i}^{\text {"goal quantity" }}\left(\frac{C_{A}}{C_{F}}\right)_{i}^{d_{i}} * \prod_{\text {Abelian factors } j}\left(\frac{e_{A}^{2}}{e_{F}^{2}}\right)_{j}^{d_{j}}\right)^{1 / d_{G}}
$$

(the notation of which I shall first explain to you later), which

- 1. is relatively simple, and

- 2. takes its biggest value for just the Standard Model Group $S(U(2) \times U(3))$.

The point then should be that this on groups defined "goal quantity", a real number, characterizes the Standard Model gauge group by pointing it out as the one that wins the game of getting the highest value for this "goal quantity". 
Here the quantities $C_{A}$ and $C_{F}$ are respectively quadratic Casimir operator values on the adjoint (irreducible) representation $A$ and on the irreducible representation $F$ with the smallest $C_{F}$ (excluding the non-faithful trivial representation) for the various simple invariant subgroup of which the group $G$ cinsidered is essentially the cross product. Therefore the ratios $C F / C_{A}$ are in fact Dynkin indices[15]. The index $i$ enumerates the simple groups in the essential cross product making up $G$ and the symbol $d_{i}$ denotes the dimensions of these simple Lie groups. Of course $d_{G}$ is the dimension of the full Lie group $G$.

Let us immediately on the following figure symbolize the result of the "game" between Lie groups with our goal quantity as the goal which is to be the largest to win. You see that the Standard Model Group $S(U(2) \times U(3))$ get the largest goal quantity, 3.91782 ... But it is very nearly followed by the next in the run, which is essentially the Standard Model Gauge group, just without the strong interaction $S U(3)$ group; that means it is in fact $U(2)$. The natural goal quantity would be to take the ratio volume of the small representation $F$ say, which is faithful to the volumeof the adjoint representation and take the $d_{G}$ th root of it to get what we culd call the linear scale ratio corresponding to the volume ratio. This most natural goal quantity would be the inverse square root of the goal quantity used in Bennett's mine first paper. On the figure you see the relation of different goal quantities expressing the same illustrated as the relation between the distances (or square of it) being identified with the goal quantity of mine and Don Bennetts old paper, while the more natural linear size of the small representation compared to the adjoint then corresponds to the time the runners needed for running a specified distance. This ratio of linear size ( determined as the $d_{G}$ root of the volume of the small representation to the adjoint is therefore put up on the figure as the times it took the runners. Apart from the squaring then the distance they reached when the goal photo was taken is identified with our old paper goal quantity. To win - i.e. to be the gauge group to be chosen by Nature to be realized - requires that you get the smallest representation in linear size. But that correponds to getting the largest value of the inverted square of this meaning the quantities discussed in our older work [2].

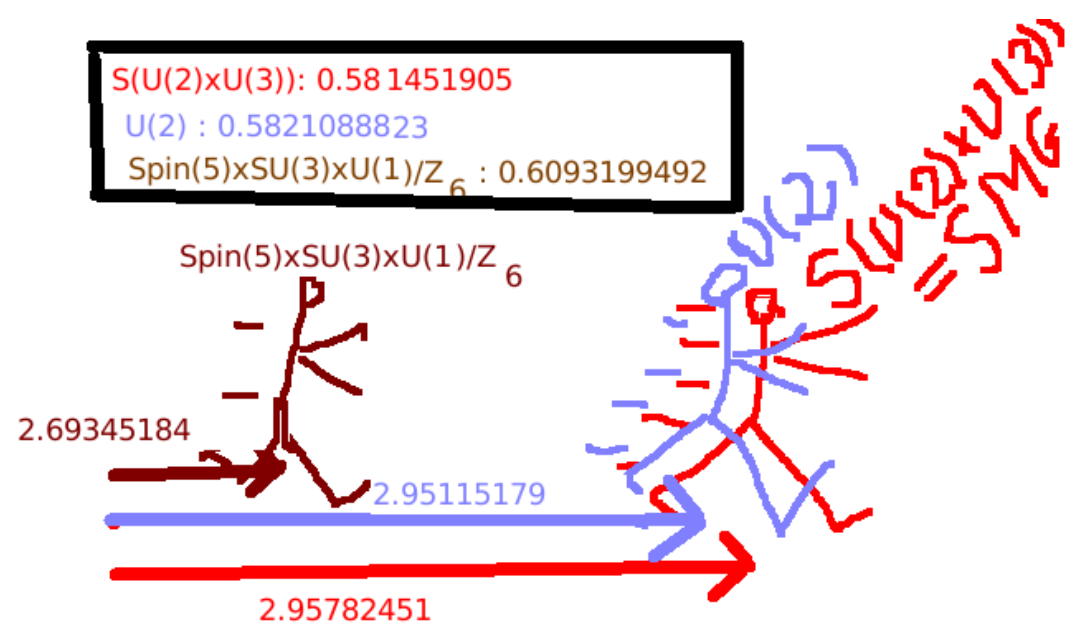

To appresiate the just described correspondance of the two equivalent ways of describing a goal quantity one should note that the numbers on the figure are related like this: $\frac{1}{\sqrt{2.95782451}}=$ 0.581451905 and e.g. $\frac{1}{\sqrt{2.69345184}}=0.6093199492$. 


\section{Correspondance group-structure $\leftrightarrow$ charge quantization rule}

Have in mind, that for our purpose of using gauge groups we just have the following equivalent ways of assigning a certain further information to a gauge theory Lie algebra:

- "group" We can assign more information to telling what the gauge Lie algebra is by telling also what is the "gauge group"

\section{is equivalent to}

- charge quantization rule Telling about certain restrictions on the representations occuring in the model (restrictions comming from that different groups with the same Lie algebra do in general not allow all the representations of the Lie algebra(or its covering group), but only a certain subset of them.

(This connection is due to: O'Raifeartaigh[3], Group Structure of Gauge theories, University Press Cambridge (1986))

\section{Outlook from the philosophy that Standard Model is the true model very far up in energy}

We have above put forward an attempt to find out, why precisely the Standard Model with its special Lie group $S(U(2) \times U(3))$ were selected by Nautre. If such a selection of indeed the Standard Model gauge Group by some principle - as above the principle of small representations - is the truth behind, then it means that the Standard Model must be the right model so far up in energy, that this Standard Model is indeed the relevant model in some very significant region of conditions. If namely, as many physicists hope for, there would appear new physics by rather little increase in the energy of the LHC say, then it would make no sense to find a characterisation of the Standard Model. The Standard Model would namely have no speial fundamental significane, but rather just be a sub-model that is part of a bigger or more extended model, which just happens to the part already discovered in 2014.

It is therefore at least suggested, that if our above characterization is relevant then the Standard Model must be a good theory over a very large energy scale, and there should be essentially no new physics at say the LHC.

But such a speculation meets severe problems:

If see saw neutrinoes needed to explain the observed neutrino oscillations and also how we could obtain an excess of matter over antimatter cosmologically, are just without gauge degrees of freedom charges, then they may not change the gauge group. Since we mainly above looked at the gauge group, we could say that an extension of the Standard Model not modifying the Standard Model Gauge Group would not be a challenge to the interest and relevance of our story of how to select the gauge group. So such a see saw neutrino extension without any extra gauge degrees of freedom being speculated could be o.k. ( But it is really not a priori o.k. w.r.t. to the idea that all the fermions with masses very small compared to the Planck scale - identified as a fundamental scale of energy - should be mass protected w.r.t. to the gauge group. The phenomenologically needed see-saw neutrinoes should namely be chargeless singlets under the Standard Model gauge group, 
and thus if the Standard Model group were selected by some great principle and thus should be the only gagueg group, the see-saw neutrinoes would have to be totally neutral and there would consequently be no way to get them mass-protected.)

Similarly a pure or minimal supersymmetry extension - which also would not change the gauge group - could be accepted without spoiling the value of the above considerations.

If we, however, not only care for the gauge group, but also for that the repsentations realized as fields in the model should be small, then the gauginos, which must belong to the adjoint representations could be claimed to not have the smallest representations, unless one takes the point of view, that one has to only take the smallest representations as far as supersymmetry allows it.

In any case I have, however, personally attempted to seek models, in which the Standard Model is everything all the way to the Planck scale or close to it except for some see saw neutrino physics. If one has this ambition of no new physics except for see saw neutrinoes and perhaps a scalar associated with it, but at least no more gauge particles at that stage, and even choose to have only minimal matter field representation like in the Standard Model, then e.g. dark matter becomes a severe problem.

Can we have dark matter in the pure Standard Model? Contrary to most other physicists, I and my collaborators C. D. Froggatt,... [36] have developped an idea for how to obtain dark matter in pure Standard Model, only extended with our (D. Bennetts and Froggatt and my own) multiple point principle[37]. This mutiple point principle - which developped from first D. Bennetts talks about commodities being fixed rather than intensive quantities - states that the universe sits just at a phase transition multiple point, wherein the coupling constants have been finetuned so as to organize, that there are several different vacuum states with very small (in older formulation of MPP the vacua should just have the same energy densities, but not a priori have small energy densities) energy densities. By introducing such a finetuning principle as this "multiple point principle" really means that one has at least the hope a priori of explaining (some of) the fine tuning problems of the physics and especially the Standard Model. If one formulates what we could think of as the "modern version" of the "multiple point principle" (MPP)[37] namely that there are several vacua and they all have very small (almost zero from high energy physics point of view) energy densities - a version for which I thank Leonard Susskind for private information -, then the usual problem of, why the cosmolgical constant is so terribly small compared to most of the various contributions, that must exist to the cosmological constant from vaious types of loop diagrams etc., has been absorbed into the multiple point assumption. This cosmological constant problem of, why the cosmological is so small is of course not really solved unless one has an explanantion for, why the several vacua all have small energy densities, but putting the assumptions together in this way means that we anyway have to have some assumption like the mutiple point one.

But if we now make this assumption of multiple point principle, then we succeeded in in fact calculate crudely the energy scale of weak interactions relative to some "fundamental" scale of energy - taken to be the Planck scale - and obtain in fact a good order of magnitude for the weak interaction energy scale[38].

\section{Can we get any hint of the Theory Beyond the Standard Model ?}

First of all one would ask one-self: Is there any mechanism, that coupld explain the principle 
of Nature selecting strongly the principle seeking the smallest faithful or at least smallest representation in the sense of having the smallest volume compared to that of the "Adjoint" representation?

Having in mind that the various fields e.g. the fermion fields that belong to these so very small representations, are shifted exceptionally little for a measured by the "Adjoint" representation somehow given shift of the group element. It means that counted in the by means of the "Adjoint" representation defined distance concept on the Lie group the fields transform exceptionally little for the gauge group selected by Nature. You could almost call the Standard Model group the most "lazy" Lie group in the sense that it varies for a given variation in the group itself the various fields as little as possible!

This story of this in some sense minimal variation under the gauge transformation of the matter fields could be explained by a model of the type that Nature at first had no gauge symmetry, but that some approximate gauge symmetry could be found by accident, and then in some way effectively become exact [21]. If one looks for an accidental approximate symmetry, there is of course the best chance to find one, when the variaton of the fields due to the symmetry operation is so small as possible. If one only varies the fields a little bit there is a much higher chance for that say the action is approximately the same after the shift as before, than if one varied the fields a lot. If you vary the fields a lot the action is expected to also vary a lot, while if the fields only are varied a bit, then also the action will from contuity vary a bit. So there is the best chance to by accident have an approximate gauge symmetry under a gauge group giving small variations of the fields than under one that leads to bigger variations of the fields. This means that our result of the Standard Model group winning the game to have the biggest value of our "goal quantity" means that it varies the fields exceptionally little and has the best value to be approximately a good symmetry just by acident. This could favour a philosophy that the fundamental theory actually is a randomly selected one and that even gauge symmetry appeared by some "Ãíxactification mechanism" working when it is there only approximately at first. Actually we with D. Første and Ninomiya have long ago proposed such an exactification mechanism[21]. The very idea of thinking of gauge symmetry as something that were there at first approximately in a random theory and then somehow comes out at the end almost by itself one could say is very much in the spirit of my long beloved project of Random Dynamics [17, 20, 21, 22, 23]. Thus we could read the message about the smallest possible faithful representations as a hint pointing towards Random Dynamics in as far as we got the suggestive explanation for our finding that it came from a random action roughly.

\subsection{Hint about Gauge Group Beyond}

There may be one hint for what could go on beyond the Standard Model, namely that we could assume that even beyond the Standard Model the gauge group is still given by our selection principle of the right gauge group to make our goal quantity maximal. It should namely be admitted that our principle of maximizing the goal quantity is not completlely unique, because we have in constructing the goal quantity balanced it so well against simple dependence on the dimensionality of the Lie group, that we have arranged that our goal quantity obeys the general rule that taking it for a cross product of a group with itself a number of times leads to the same goal quantity as for the cross product factors. That is to say we had arranged

$$
g q(G)=g q(G \times G \times \cdots \times G) .
$$


Here we denoted the goal quantity for a group $\mathrm{G}$ say as $g q(G)$.

Thus it were not totally correct that just the Standard Model group $S(U(2) \times U(3))$ were uniquely pointed out by our goal quantity. Rather it could equally well have been the cross product of any number of standard model groups. I.e. it could equally well have been any group like $S(U(2) \times U(3)) \times S(U(2) \times U(3)) \times \cdots S(U(2) \times U(3)) \times S(U(2) \times U(3))$ that could be claimed to be "the" selceted one. This ambiguity in our selction after all gives us the possibility to have a group with a larger dimensionality than the 12 of the Standard Model group, so that there could be a chance to take it as an extension of the Standard Model group. we could namely propose one of these cross products of the Standard Model group with itself a number of times. The standard model group itself can be found as a subgroup of such a cross product of standard model groups with itself in a few different ways. It can for example be found as what we call the diagonal subgroup of the cross product group. In a group that is a cross product of say a group $G$ with itself a number of times $G \times G \times \cdots \times G$ the elements in it are of course of the form $\left(g_{1}, g_{2}, \ldots g_{n}\right)$ where the $g_{i} \in G$. The diagonal subgroup is the group consisting of just those elements in $G \times G \times \cdots \times G$ for which all the component elements $g_{i}$ are equal to each other, i.e. $g_{1}=g_{2}=\cdots=g_{n}$. Long tme ago we worked with a model which were called AntiGUT model - in contrast of course to usual grand unified theory[24] - and it that the high energy or original ((anti)unifying) group were such a cross product of the Standard Model group with itself a number of times - namely one factor in the cross product for each family of quarks and leptons $-S(U(2) \times U(3)) \times S(U(2) \times U(3)) \times$ $\cdots S(U(2) \times U(3)) \times S(U(2) \times U(3))$. In this AntiGUT model each family of quarks and leptons would so to speak obtain its own set of gauge particles.

Here we just stress that strictly speaking our small representation principle has as its only possible extension of the Standard Model to a larger gauge group the possibilty of using this kind of antiGUT type theories.

Actually antiGUT has several advantages: 1) Contrary to simple GUT theories the extension can contribute to the small hierarchy, meaning it may help in explaining that there are orders of magnitude differences between various quarks and leptons.

2) We once used it combined with what we would now consider "multiple point principle" to fit the fine structure constants using the number of families - which were at that time not knownas a parameter and in this way we PREdicted the number of families to be three form MPP and AntiGUT background gauge theory.[]

\section{Resume Conclusion}

- We have defined a measure for the size of representation $r$ relative to that of the adjoint representation for the same group $G$, so that we even can compare "sizes" of representations of different groups.

- The concept of this "adjoint" representation is the straightforward concept - the representation on the Lie algebra - for the non-abelian part of the Lie group, but has to be defined by us for the Abelian part.

- Including in the counting only faithful representations (which are the ones representing every element of the group by a seperate element) we seek the very smallest representation among 
all the different groups; then the Lie group having this very smallest faithful representation is just the Standard Model gauge group.

\section{7. appendix}

For calculating our "goal quantity $\hat{A} t$ 'Ât' for various groups one has in addition to evaluate factor connected with the abelian invariant subgroups - something done by considering how strongly the associated restriction relations (which essentially is the charge quantization rule) restricts the representations - the factor associated with the simple nonabelian invariant subgroupos. The latter factor is obtained by first evaluating for each simple invariant non-abelian subgroup the ratio of the quadratic Casimir for respectively the adjoint representation and that faithful representation of the simple non-abelian group that has the smallest quadratic Casimir. In the reference[2] we constructed a table of ratios $C_{A} / C_{F}$ for the quadratic Casimir $C_{A}$ for the adjoint representation $A$ to that $C_{F}$ for the faithful repsentation $F$ with $C_{F}$ minmial among faithful representations )really it is only the trivial representation that is excluded from being the $F$ when we talk about simple Lie algebras). Here we used the terminology of simple a bit wrongly in as far as we called even the Lie groups which are not simple, if they have simple Lie algebra. For the $S O(N)$ groups - $B_{n}$ and $D_{n}$ for odd and even $N$ respectively - the faithful representation with the smallest quadratic Casimir may depending on the rank $n$ or the dimension $N$ be a spinor representation or a vector representation. Therefore we give in the table below from reference [2] two cases for these $S O(N)$ Lie groups.

Our Ratio of Adjoint to "smallest" Quadratic Casimirs $C_{A} / C_{F}$, for faithful repr.

$$
\begin{aligned}
\left.\frac{C_{A}}{C_{F}}\right|_{A_{n}} & =\frac{2(n+1)^{2}}{n(n+2)}=\frac{2(n+1)^{2}}{(n+1)^{2}-1}=\frac{2}{1-\frac{1}{(n+1)^{2}}} \\
\left.\frac{C_{A}}{C_{F} \text { vector }}\right|_{B_{n}} & =\frac{2 n-1}{n}=2-\frac{1}{n} \\
\left.\frac{C_{A}}{C_{F} \text { spinor }}\right|_{B_{n}} & =\frac{2 n-1}{\frac{2 n^{2}+n}{8}}=\frac{16 n-8}{n(2 n+1)} \\
\left.\frac{C_{A}}{C_{F}}\right|_{C_{n}} & =\frac{n+1}{n / 2+1 / 4}=\frac{4(n+1)}{2 n+1} \\
\left.\frac{C_{A}}{C_{F} \text { vector }}\right|_{D_{n}} & =\frac{2(n-1)}{n-1 / 2}=\frac{4(n-1)}{2 n-1} \\
\left.\frac{C_{A}}{C_{F} \text { spinor }}\right|_{D_{n}} & =\frac{2(n-1)}{\frac{2 n^{2}-n}{8}}=\frac{16(n-1)}{n(2 n-1)} \\
\left.\frac{C_{A}}{C_{F}}\right|_{G_{2}} & =\frac{4}{2}=2 \\
\left.\frac{C_{A}}{C_{F}}\right|_{F_{4}} & =\frac{9}{6}=\frac{3}{2} \\
\left.\frac{C_{A}}{C_{F}}\right|_{E_{6}} & =\frac{12}{\frac{26}{3}}=\frac{18}{13} \\
\left.\frac{C_{A}}{C_{F}}\right|_{E_{7}} & =\frac{18}{\frac{57}{4}}=\frac{72}{57}=\frac{24}{19}
\end{aligned}
$$




$$
\left.\frac{C_{A}}{C_{F}}\right|_{E_{8}}=\frac{30}{30}=1
$$

The notation is this table is the mathematical classification of the Lie algebras:

$$
\begin{aligned}
& A_{n}=S U(n+1) \\
& B_{n}=S O(2 n+1) \\
& C_{n} \quad(\text { symplecticLiegroup }) \\
& D_{n}=S O(2 n)
\end{aligned}
$$

and the Lie groups $G_{2}, E_{n}, F_{4}$ are the special Lie groups.

\subsection{An example}

As an example you may use the table to obtain the goal quantity for the Standard Model gauge group:

The two simple non-abelian groups for which we need the $C_{A} / C_{F}$ ratio are $S U(2)=A_{1}$ giving $\left(C_{A} / C_{F}\right)_{S U(2)}=8 / 3$ and $S U(3)=A_{2}$ which gives $\left(C_{A} / C_{F}\right)_{S U(3)}=9 / 4$. These ratios mean the squares of the scaling factor in the group or representation manifolds of those dimensions corresponding to the simple grouo in question of the adjoint compared to the $F$ representation ) characterized as smallest $C_{F}$ for any faithfull representation of the simple group. Were it not for the factor involving the abelian part and the division out of a subgroup of the center, we would obtain the factor - from the simple nonabelian - $(8 / 3)^{3 / 2} *(9 / 4)^{8 / 2}$ for the ratio of the volume of the adjoint representation manifold to the one for the to the $F$ representations corresponding. The rule to multiply this volume ratio by the number 6 comming from the quantization restriction only allowing crudely speakung one out of 6 combinations. Now to avoid as we asked for in the article a too strong dependence on the dimesnion of the group, we decided to take the the $d_{G}$ th root of the volume ratio. So if we decide to look for a (linear) size ratio of the $F$ one (the small one) to the adjoint our goal quatity - now to be minmized to win -

$$
\text { goal-quantity }=\frac{1}{\left((8 / 3)^{3 / 2} *(9 / 4)^{8 / 2} * 6\right)^{1 / 12}} .
$$

To avoid too much confusion due to that we used in this article a goal-quantity that is the inverse square root of the one used in previous papers and even inthe figure here let me translate this expression to the notation of the earlier papers

$$
\text { earlier }- \text { goal }- \text { quantity }=\left((8 / 3)^{3 / 2} *(9 / 4)^{8 / 2} * 6\right)^{2 / 12} .
$$

\section{References}

[1] H. B. Nielsen, "Dimension Four Wins the Same Game as the Standard Model Group," arXiv:1304.6051 [hep-ph].

[2] Don Bennett and H. B. Nielsen, "Seeking...”, Contribution to the workshop "Beyond the Standard Models", Bled 2011. 
[3] O’Raifeartaigh, Group Structure of Gauge theories,University Press Cambridge (1986))

[4] H. B. Nielsen, "Small Representation Principle", Contribution to the workshop "Beyond the Standard Models", Bled 2013.

[5] Froggatt, C.D. et al. "Remarkable coincidence for the top Yukawa coupling and an approximately massless bound state" - Phys.Rev. D80 (2009) 034033 arXiv:0811.2089 [hep-ph]; Richard, Jean-Marc "About the stability of the dodecatoplet" Few Body Syst. 45 (2009) 65-70 arXiv:0811.2711 [hep-ph] Nielsen, Holger Bech “ PREdicted the Higgs Mass” arXiv:1212.5716 [hep-ph] Froggatt, C.D. et al. “ Remarkable coincidence for the top Yukawa coupling and an approximately massless bound state" Phys.Rev. D80 (2009) 034033 arXiv:0811.2089 [hep-ph] Nielsen, Holger Bech, “ PREdicted the Higgs Mass" arXiv:1212.5716 [hep-ph] Das, C.R. et al. "New Bound States of Top and Beauty Quarks at the Tevatron and LHC”, arXiv:0908.4514 [hep-ph] Das, C.R. et al. "New Bound States of Heavy Quarks at LHC and Tevatron” Int.J.Mod.Phys. A26 (2011) 2503-2521 arXiv:0812.0828 [hep-ph]

[6] Max Tegmark, “On the dimensionality of space time”, Class. Quantum Grav. 14, 1.69 - 1.75 (1997).

[7] Ehrenfest,P., 1917 Proc. Amsterdam Acad. 20200

[8] Gordon Kane : http://particle-theory.physics.lsa.umich.edu/kane/modern.html

[9] Norma Mankoc et al. see many contributions to the present and previuos Bled Proceedings.

[10] H. B. Nielsen and N. Brene, "Spontaneous Emergence Of Gauge Symmetry," IN *KRAKOW 1987, PROCEEDINGS, SKYRMIONS AND ANOMALIES*, 493-498 AND COPENHAGEN UNIV. NBI-HE-87-28 (87,REC.JUN.) 6p H. B. Nielsen and N. Brene, "Skewness Of The Standard Model: Possible Implications,” Physicalia Magazine, The Gardener of Eden, 12 (1990) 157; NBI-HE-89-38; H. B. Nielsen and N. Brene, "What Is Special About The Group Of The Standard Model?," Phys. Lett. B 223 (1989) 399.

[11] H.B. Nielsen, S.E. Rugh and C. Surlykke, Seeking Inspiration from the Standard Model in Order to Go Beyond It, Proc. of Conference held on Korfu (1992)

[12] C. D. Froggatt, R. Nevzorov and H. B. Nielsen, arXiv:1103.2146 [hep-ph]; C. D. Froggatt, R. Nevzorov and H. B. Nielsen, Nucl. Phys. B 743 (2006) 133; C. D. Froggatt, L. V. Laperashvili, R. Nevzorov and H. B. Nielsen, Phys. Atom. Nucl. 67 (2004) 582 [arXiv:hep-ph/0310127]. SUSY11, Fermilab, Batavia IL, USA, August 28 âĂ $\breve{T}$ September 2, 2011 âĂŞ p. 2/16

[13] Oliver, David (2004). "The shaggy steed of physics: mathematical beauty in the physical world." Springer. p. 81. ISBN 978-0-387-40307-6. Humphreys, James E. (1978). "Introduction to Lie Algebras and Representation Theory." Graduate Texts in Mathematics 9 (Second printing, revised ed.). New York: Springer-Verlag. ISBN 0-387-90053-5. Jacobson, Nathan (1979). "Lie algebras." Dover Publications. pp. 243ÃćÂĂÂŞ249. ISBN 0-486-63832-4.

[14] Millikan, Robert Andrews (1917). The Electron: Its Isolation and Measurements and the Determination of Some of its Properties. The University of Chicago Press.

[15] Philippe Di Francesco, Pierre Mathieu, David SÃl'nÃl'chal, Conformal Field Theory, 1997 Springer-Verlag New York, ISBN 0-387-94785-X

[16] C.D. Froggatt and H.B. Nielsen“Why do we have parity violation?” arXiv:hep-ph/9906466v1, 23 Jun 1999. 
[17] RANDOM DYNAMICS: H. B. Nielsen, "Dual Strings,", "Fundamentals of quark models", In: Proc. of the Seventeenth Scott. Univ. Summer School in Physics, St. Andrews, august 1976. I.M. Barbour and A.T. Davies(eds.), Univ. of Glasgow , 465-547 (publ. by the Scott.Univ. Summer School in Physics, 1977) (CITATION = NBI-HE-74-15;)

[18] C. D. Froggatt, H.B. Nielsen, Y. Takanishi "Neutrino Oscillations in Extended Anti-GUT Model" Talk given at the Second Tropical Workshop on Particle Physics and Cosmology, San Juan, Puerto Rico May 2000. arXiv: hep-ph/0011168v1

[19] Patterson, Elizabeth C. (1970). John Dalton and the Atomic Theory. Garden City, New York: Anchor.

[20] H.B. Nielsen, Har vi brug for fundamentale naturlove(in Danish) (meaning:"Do we need laws of Nature?”) Gamma 36 page 3-16, 1978 (1. part) and Gamma 37 page 35-46, 1978 (2. part)

H.B. Nielsen and C. D. Froggatt, "Statistical Analysis of quark and lepton masses", Nucl. Phys. B164(1979) 114 - 140.

[21] D. Førster, H.B. Nielsen, and M. Ninomiya, "Dynamical stability of local gauge symmetry. Creation of light from chaos.” Phys. Lett. B94(1980) 135 -140

[22] H.B. Nielsen, Lecture notes in Physics 181, "Gauge Theories of the Eighties" In: Proc. of the Arctic School of Physics 1982, Akaeslompolo, Finland, Aug. 1982. R. Raitio and J. Lindfors(eds.). Springer, Berlin, 1983,p. 288-354.

H.B. Nielsen, D.L. Bennett and N. Brene: "The random dynamics project from fundamental to human physics". In: Recent developments in quantum field theory. J. Ambjoern, B.J. Durhuus and J.L. Petersen(eds.), Elsvier Sci.Publ. B.V., 1985, pp. 263-351

[23] See the "home page of Random Dynamics": http://www.nbi.dk/ kleppe/random/qa/qa.html

[24] Ross, G. (1984). Grand Unified Theories. Westview Press. ISBN 978-0-8053-6968-7.

Georgi, H.; Glashow, S.L. (1974). "Unity of All Elementary Particle Forces". Physical Review Letters 32: 438 441. Bibcode:1974PhRvL..32..438G. doi:10.1103/PhysRevLett.32.438.

Pati, J.; Salam, A. (1974). "Lepton Number as the Fourth Color". Physical Review D 10: 275289. Bibcode:1974PhRvD..10..275P. doi:10.1103/PhysRevD.10.275.

Buras, A.J.; Ellis, J.; Gaillard, M.K.; Nanopoulos, D.V. (1978). "Aspects of the grand unification of strong, weak and electromagnetic interactions". Nuclear Physics B 135 (1): 66G 92.

Bibcode:1978NuPhB.135...66B. doi:10.1016/0550-3213(78)90214-6. Retrieved 2011-03-21.

Nanopoulos, D.V. (1979). "Protons Are Not Forever". Orbis Scientiae 1: 91. Harvard Preprint HUTP-78/A062.

Ellis, J. (2002). "Physics gets physical". Nature 415 (6875): 957. Bibcode:2002Natur.415..957E. doi: $10.1038 / 415957 \mathrm{~b}$.

Ross, G. (1984). Grand Unified Theories. Westview Press. ISBN 978-0-8053-6968-7.

Hawking, S.W. (1996). A Brief History of Time: The Updated and Expanded Edition. (2nd ed.). Bantam Books. p. XXX. ISBN 0-553-38016-8.

[25] H.B. Nielsen and D. L. Bennett, "The Gauge Glass: A short review", Elaborated version of talk at the Conf. on Disordered Systems, Copenhagen, September 1984. Nordita preprint 85/23.

[26] see e.g. John Baez: http://math.ucr.edu/home/baez/renormalizability.html, November 2006

[27] P. Candelas, Gary T. Horowitch, Andrew Strominger, and Edward Witten, "Vacuum configurations for superstrings",NFS-ITP-84-170. 
[28] Candelas...Witten, Nuclear Physics B 258: 46 \% G \% 74, Bibcode:1985NuPhB.258...46C, doi:10.1016/0550-3213(85)90602-9

[29] See e.g. John M. Pierre, "Superstrings Extra dimensions" http://www.sukidog.com/jpierre/strings/extradim.htm

[30] P.H. Damgaard, N. Kawamoto, K. Shigemoto: Phys. Rev. Lett.53, 2211 (1984)

[31] A J Macfarlane and Hendryk Pfeiffer, J. Phys. A: Math. Gen. 36 (2003) 2305 Ãŕ̂̂£鬼 2002232317 PII: S0305-4470(03)56335-1 Representations of the exceptional and other Lie algebras with integral eigenvalues of the Casimir operator

[32] T. van Ritbergen, A. N. Shellekens, J. A. M. Vermaseren, UM-TH-98-01 NIKHEF-98-004 Group theory factors for Feynman ... www.nikhef.nl/ form/maindir/oldversions/. ../packages/.../color.ps

[33] Physics Letters B Volume 208, Issue 2, 14 July 1988, Pages 275-280, http://arxiv.org/abs/hep-ph/9311321

http://arxiv.org/abs/hep-ph/9607341

Physics Letters B Volume 178, Issues 2-3, 2 October 1986, Pages 179-186

H.B. Nielsen and N. Brene, Gauge Glass, Proc. of the XVIII International Symposium on the Theory of Elementary Particles, Ahrenshoop, 1985 (Institut fur Hochenergiphysik, Akad. der Wissenschaften der DDR, Berlin-Zeuthen, 1985);

[34] Lehto, M., Nielsen H. B., and Ninomiya, M. (1986). Pregeometric quantum lattice: A general discussion. Nuclear Physics B, 272, 213-227. Lehto, M., Nielsen H. B., and Ninomiya, M. (1986). Diffeomorphism symmetry in simplicial quantum gravity. Nuclear Physics B, 272, 228-252. Lehto, M., Nielsen H. B., and Ninomiya, M. (1989). Time translational symmetry. Physics Letters B, 219, 87-91.

[35] M. Lehto, H. B. Nielsen, and Masao Ninomiya "A correlation decay theorem at high temperature" Comm. Math. Phys. Volume 93, Number 4 (1984), 483-493.

[36] C. D. Froggatt and H. B. Nielsen, Tunguska Dark Matter Ball arXiv:1403.7177v2[hep-ph] and ealier works

[37] D. L. Bennett and H. B. Nielsen, âĂIJPredictions for nonAbe lian fine structure constants from multicriticality,âĂİ Int. J. Mod. P hys. A 9 (1994) 5155 [arXiv:hep-ph/9311321]. D. L. Bennett, âĂIJMultiple point criticality, nonlocality an d fine tun- ing in fundamental physics: Predictions for gauge coupling constants gives alpha**(-1) = $136.8+-9$, âĂ İ arXiv:hep-ph/9607341. (

[38] Froggatt, CD, Nielsen, HB and Laperashvili, LV 2005, 'Hierarchy-problem and a bound state of $6 \mathrm{t}$ and 6 anti- t' International Journal of Modern Physics A, vol 20, ... 